\title{
Los Tres Planos de la Creación Artística de Eduardo Barrios
}

La verdad sicológica ha sido negada o puesta en duda repetidamen. te con respecto a la obra de Eduardo Barrios. El objeto de este artículo es llamar la atención sobre el hecho de que en general la crítica no ha visto claramente este aspecto de la creación artística en el novelista chileno. Vamos a demostrarlo al examinar la teoría del arte de Barrios y al verificar dicha teoría en su obra.

Ante todo, tropezamos con dificultades de terminología. ${ }^{1}$ Veamos un ejemplo. Leon Edel, una de las autoridades de la novela sicológica en este momento, hace equivaler las expresiones "subjetivo" y "sicológico".2 Enrique Anderson Imbert, por su parte, dice: "Tanto hemos usado a propósito de Barrios ell concepto 'psicológico' que conviene advertir que sus novelas más que psicológicas, son subjetivas". ${ }^{3}$ Es evidente que Anderson Imbert, a diferencia de Edel, opone sicológico a subjetivo, y al hacerlo, acerca aquel concepto al de objetividad.

En este punto el problema se hace más de fondo: si nuestra interpretación del texto de Anderson Imbert es acertada, la "objetividad" en la creación novelística consistiría, pues, entre otras cosas, en la capacidad del autor de crear y analizar personajes diferentes a él mismo. Aquí no vamos a ocuparnos del problama de si es posible a no que el autor

1 Ned J. Davison, en su tesis doctoral Psycbological Values in the Works of Eduardo Barrios, dice: "There has been some confusion whether Barrios is a 'psychological' novelist. That he is such a novelist is the implicit assumption of this study, and I shall not attempt to demonstrate in any detail the validity of such an assumption since most of the confusion arises out of conflicting definitions of the term "psychological novel" (University of California, Los Angeles, 1957 , p. 11).

i The Modern Psycbological Novel (New York: Grove Press, 1955), pp. $122,136,138$ y 140.

1957) Historia de la literatura bispanoamericana (2* ed., México: F. C. E., 
salga de la propia conciencia para objetivarse en sus personajes. Limitémonos a aclarar que lo que nosctros entendemos por "verdad sicológica" en este estudio. sobre Barrios no es solamente la verosimilitud y fuerza con que nos impresionan los personajes (que en este sentido podrían ser todos réplicas de su autor), sino la adecuación de las siquis de los personajes a las que pueden analizarse en la vida real, con toda su variedad. Es decir, que nosotros también relacionaremos los conceptos de "sicológico" y "objetivo".

Ahora bien, en el segundo juicio que hemos transcripto nos parece que se insinúa una vuelta a opiniones anteriores que acentuaban lo subjetivo-autobiográfico en la creación de Barrios. Lo cual se reafirma con lo que Anderson dice a continuación: "Lo cierto es que Barrios usa convenciones narrativas no siempre verosímiles y a veces increíbles desde un punto de vista rigurosamente psicológico" (Ibid., p. 353). Y al hablar de obras en particular, y en cuanto al análisis sicológico, afirma que Un perdido es "más penetrante" que $E l$ niño que enloqueció de amor. Esta opinión, aunque no necesariamente igual en todos sus aspectos y fundamentos, es parecida a la de varios otros críticos, especialmente en lo que toca a la famosa novela corta de Barrios. Nos limitaremos a citar a los últimos: Alberto Zum-Felde, en su extensa obra de I959, afirma que la pasión del "niño" es inverosímill, y los profesores santiaguinos Julio Orlandi y Alejandro Ramírez, en su libro sobre Barrios del año I960, hablan de "esta historia sencilla y de buscada incoherencia". 5 Con respecto a opiniones anteriores en este sentido, el crítico chileno Milton Rossel, a la vez que se opone a ellas, sostiene que existe "la verosimilitud indispensable para que la ficción novelesca sea lograda". ${ }^{6}$

La posición de Milton Rossel apunta a la que se encuentra en una de las críticas sobre Barrios que apreciamos más, la de Arturo Torres Ríoseco. En su influyente libro sobre Grandes novelistas de la América bispana, Torres Ríoseco afirma la "observación exacta de la realidad" en Barrios (II, 27) - con lo que estamos completamente de acuerdo-, y asimismo la "penetración" y la "seguridad magistral" del narrador (II, 26). Pero poco más adelante insiste en ciertas salvedades dubitativas

* Indice crítico de la literatura bispanoamericana, II, La narrativa (México: Ed. Guaranía, 1959), p. 216.

5 Eduardo Barrios. Obras -Estilo-Técnica (Santiago de Chile: Ed, del Pacífico, 1960), p. 27.

- En su comentario a la sexta edición de El niño que enloqueció de amor, Atened, $\mathrm{n}^{\circ} 174$ (1939), pp. 427-429. 
de la página anterior, diciendo que "para un psicólogo acaso no exista en esta obrita la verdad científica" (II, 27), y a continuación trata de justificar su juicio, así como de dejar a salvo el mérito artístico de Barrios, con una cita de este último: la definición del arte que se encuentra en $Y$ la vida sigue, como "una ficción que sirve para comunicar, no la verdad misma, sino la emoción de la verdad".

Nosotros también creemos, junto con Torres Ríoseco y con Barrios -y siguiendo a Maupassant- que sin la "emoción de la verdad" fracasa el arte. Pero también crecmos que dicha observación no basta para obtener una imagen clara y exacta del proceso creador en el novelista chileno, y que es necesario hacer un análisis más detenido de su definición del atte, tener además en cuenta otras declaraciones de carácter teórico no menos importantes, y observar lo que se desprende de repetidas anécdotas, así como de persistentes actitudes de Barrios con respecto a sus obras a lo largo de toda su vida.

Veamos. Ante todo, en el texto sobre el arte que hemos transcripto no hace falta entender que Barrios le quite importancia a la verdad, con tal de conseguir una apariencia, o impresión, o "emoción" de la verdad. En el párrafo que sigue dice: "Acerca de mi definición del arte, no creo necesario insistir. Cuando más, pido fijarse en que digo comunicar y no exprestr [cursiva en el original]. La expresión lisa y llana, por exacta y poderosa que sea, pertenece a la ciencia: comunicar y aun contagiar es misión del artista" (ibid., p. 87). Mantengámonos alerta sobre la terminología: aquí nuestro autor usa varios términos en sentido opuesto a los pensadores vinculados con el idealismo y expresionismo alemán. Su palabra "comunicar" está relacionada con la idea de comunión —unión intima y total-mientras que "expresar" está usado en el sentido corriente del término, que vale tanto como "decir", "informar". Hasta aquí la verdad no deja de ser importante para Barrios, pero no es operativa por sí sola, y el arte no debe servirse de un procedimiento científico de expresión.

Hay otro escrito de Barrios sobre estos temas, que nos parece revelador de la importancia que le da a la verdad científica, a la vez que insiste en la necesidad de hacerla pasar inadvertida en la creación artística. Se trata del prólogo a una colección de cuentos de Alfonso Hernández Catá, en el cual Barrios elogia mucho a Hernández y, de paso, deja a veces sentado su propio credo artístico y revela otros hechos no menos significativos. Vuelve a definir el arte y el proceso creador:

7 Buenos Aires: Tor, 1925, p. 87. 
"...el arte no es otra cosa que revelación, en su quid radical [es decir, que la verdad está en la raíz]; y luego, milagro comunicativo en su cumplimiento". A continuación habla del conocimiento que se transforma en cultura, y añade: "...esta cultura, sin remedio, se vuelve arte en él [el artista], porque cuanto hay de objetivo en ella se subjetiviza al ser captado por el temperamento creador. ...Y este disfraz de la ciencia es necesario, indispensable al artista. Su obra ha de hallarse tan saturada de subjetivismo, que luego, en la serenidad suprema de la forma externamente objetiva, se nos presente como un espontáneo millagro, como la verdad sencilla de un niño, como la ingenuidad de un simple". 8 Esto es, según Barrios, que a partir de la materia de verdad con la que trabajará el artista, la creación da dos pasos: el primero de "subjetivizar", personalizar, hacer desaparecer toda la prosa y desapego de la ciencia, de la observación e intuición objetiva; el segundo y final, de dominar lo subjetivo y personal e imponerle una actitud objetiva y un desapego en el plano exterior de la forma artística. De todo lo cual resulta la más celosa ocultación de los materiales, fórmulas y trasmutaciones.

A este último paso vuelve a referirse unas líneas más abajo, en la misma página, cuando dice "el drama del concepto crucificando el temperamento", que nos recuerda lo que en su ensayo sobre clasicismo André Gide llamaba "le romantisme dompté" - el romanticismo domado, subyugado-, que debe existir para que la obra clásica sea fuerte y be$11 \mathrm{a} .^{9}$

Ya tenemos los tres planos de la creación artística bien delineados: uno primario, totalmente encubierto y difícil de penetrar, de rigurosa observación e intuición objetiva; un segundo plano intermedio, que se transparenta desde fuera, de identificación subjetiva y emocional con el personaje; finalmente y cubriendo por completo a los otros dos, un tercer plano de serenidad y dominio de la materia, de economía y exactitud expresiva, de plasmación de la palabra que infunde realidad al sentimiento.

Hasta aquí llega la teoría artística en los escritos de Barrios. Aparte de alguno que otro reportaje que cubre aspectos parciales de la creación, hasta ahora no hay más escritos sobre la materia. $\mathrm{O}$, si los hubiere, no están a la mano.

8 Alfonso Hernández Catá, Sus mejores cuentos (Santiago de Chile: Nascimento, 1936), p. 9 [Prólogo de Eduardo Barrios].

9 Morceaux choisis ( $27^{\mathrm{a}}$ edición, París: Gallimard, 1921), p. 93. 
Ahora bien: ¿corresponde este credo del escritor chileno a su actitud y a lo que ha hecho en la obra misma? Con otras palabras: ¿encontramos en la obra de Barrios ese plano que llamamos primario por servir de base y núcleo a los demás - el plano de la verdad sicológica objetiva? En busca de respuesta, llamaremos la atención sobre algunos aspectos reveladores, en orden cronológico, a partir de los comienzos del escritor, hasta sus primeras obras novelísticas. Como precisamente a éstas se les ha atribuido con más frecuencia un supuesto carácter subjetivo, o autobiográfico, cuando no se les ha imputado una falta de verosimilitud, la verificación de esa base objetiva $y$ hasta científica en las mismas -especialmente en El niño que enloqueció de amor será el argumento más contundente en favor de nuestra tesis.

Nos referiremos primero al libro inicial de nuestro autor, una obra imperfecta, de juventud, que está muy lejos de las excelencias de sus novelas posteriores, pero que apunta desde un comienzo a su pretensión y esfuerzo por asir la verdad objetiva en los caracteres y en la vida, y presentarla con toda fidelidad. Del natural se llama el libro; la elipsis del título sugiere, y de ese modo recalca, la idea de copia fiel. ${ }^{10}$ Tenemos aquí al escritor naturalista, un naturalista tardío y a medias, pero no por ello menos imbuido. El prólogo mismo del libro confirma una actitud -o por lo menos una aspiración-eminentemente objetiva y científica, a la vez que una vocación sociológica y de reformador social. Esta actitud persiste durante el período teatral que termina en I9I6; luego desaparece de la superficie, pero creemos que un estudio atento revelará que el observador, crítico y reformador de la sociedad se encuentra siempre, más o menos latente, en casi toda la obra de Barrios, no sólo en $U n$ perdido, sino en El bermano asno, en los cuentos, y en sus últimas novelas. ${ }^{11}$

10 La idea implícita y hasta los términos del primer título de Barrios habian estado de moda en España una generación antes. Se encuentra el mismo tí. tulo Del natural en un escritor secundario, E. G. Gamero (Jaén, 1880). En 1879 Narciso Oller y Moragas publicó Croquís del natural. En 1882 José Alcázar Hernández publicó en La revista dé España, "Los hombres de bien-Estudios del natural" (vol. 86, pp. 66), y "Matina-Estudio del natural" (vol. 89, p. 262). Estos nombres nos los facilitó el profesor Walter T. Pattison; se trata tan sólo de una muestra, pues hay gran número de títulos semejantes en ese período de la literatura de España. También podrían tenerse en cuenta conceptos relacionados, como el que se encuentra por ejemplo en el títula de la misma época des Palacio Valdés, Aguas fuertes (1884) - colección de cuentos que sugieten más a Dickens que a los realistas franceses (a quienes trató de seguir más tarde). Por esos años Pereda ya contribuía al realismo español- que nada tenía que ver con el de Zola (cuyas grandes obras empezaron en 1871 con Les Rougon-Macquart) $-y$ publicó Bocetos al temple en 1876 y Esbozos y rasguños en 1881.

1,1 El desarrollo de este punto merece un estudio especial, que esperamos 
Que no se entienda que señalamos más de la cuenta este interés sociológico. Aun cuando nunca desaparece del todo, muy pronto cede el primer lugar al interés sicológico. Este cambio ocurre paralela o conjuntamente con el desencanto del teatro, el cual se debió, según Barrios, al choque de la integridad artística del autor con la irreductible vanidad de las actrices. ${ }^{1 / 2}$

Otra vez el escritor se entenderá a solas con la pluma y el papel, y lo más notable de esta vuelta de Barrios es que su penetración sicológica y su poder comunicativo se manifiestan en toda su fuerza desde un principio, en las breves páginas de El niño que enlóquéció de amor. En esta novela corta ya encontramos, llevado a su perfección, el doble proceso, que describirá más tarde, de identificación subjetiva y emocional con el protagonista primeramente - "subjetivización"-, seguida del perfeccionamiento "en la serenidad suprema [con sus propias palabras] de la forma externamente objetiva", con lo cual establece definitivamente, para de alli en adelante, los tres planos en que se estructura su creación artística. Además, "subjetivización" y "disfraz de la ciencia" aparecen ya como dos aspectos relacionados de la misma transformación artística. $\mathrm{Y}$ un estudio suficientemente extenso y detenido descubrirá una singular y sorprendente exactitud y acumulación de detalles de valor científico, hasta tal punto que se hace difícil imaginar que tantos aciertos puedan ser resultado del poder de observación e intuición sicológica de un novelista. Aquí nos limitaremos a algún ejemplo ilustrativo.

Es sabida la insistencia con que Barrios afirmó y repitió, desde I9I5 hasta la fecha, el antecedente autobiográfico de la pasión de su "niño". Observemos, de paso, que con ello no acentuaba un carácter subjetivo

poder cumplir pronto. Como ejemplo, séanos permitido mencionar por ahora, en Gran señor y rájadiablos, su concepción histórica y política del patrón rural a lo caudillo y su papel en la sociedad (Santiago de Chile: Nascimento [ed. Círculo Literario], 1948, pp. 267-268), y señalar sus frecuentes referencias a la teligión en Chile (pp. 54, 59, 102,116,139, 148, 160 y ss.; 234, 246, 325, 344, $346,382$ y ss.; $395,396,401$ y ss.; $413,486,493)$.

12 En agosto de 1960 tuvimos el honor de ser recibidos por don Eduardo Barrios en su residencia de Av. F. Bilbao 1966, en Santiago de Chile. En una de las visitas nos nartó una anécdota vinculada a la representación de su último drama Vivir (1916), en la cual la primera actriz, a la que correspondía el papel de abuela, pretendía que se hicieran ciertos cambios para favorecerla. "Entonces me desengañé del teatro - dijo don Eduardo Barrios-, porque vi que existía esa vanidad de los actores. El autor debe someterse a una serie de requerimientos -pequeñeces de los actores, para lucirse ellos en desmedro de la honradez artística del autor". $Y$ en $Y$ la vida sigue... decía: "Si cultivo de preferencia la novela, es porque en ella entran todos los géneros: el episodio no es otra cosa que el cuento; el diálogo coge del teatro la palpitación viva el calor del movimiento, y con la ventaja de hacerlo en voz queda..." (p.88). 
de su obra o una falta de independencia del "niño", sino que su protesta y revelación estaba encaminada a probar la verosimilitud y aún la verdad de su personaje. Cuando lo visitamos en su residencia de Santiago, en I960, volvió sobre ell tema con las siguientes palabras: "...me pasó a mí, y no me atrevía a publicarlo por aquello que después pasó, que algunos no creerían en la verosimilitud. ."13 Observemos ahora un detalle y transformación importante en el personaje, que revela su independencia con respecto al autor. Este fue un chico gordo; su condiscípulo limeño Ventura García Calderón, cuando adulto, todavía le ponía en las cartas "querido gordo", y Barrios mismo, en $Y$ la vida sigue..., nos dice que "los gordos son siempre buena gente. Será superstición mía; pero yo, hoy casi flaco, temo deber mi dosis de bondad a mi gorda infancia..." (p. 84). En la novela podría no haberse tocado este punto, pero no ocurre asi: ". . dice mi abuela que soy una pobre criatura, que estoy flaco y paliducho, que tengo las piernas como palillos..."14 $\mathrm{Y}$ las cosas no ocurren por casualidad en Barrios: Lucho, de Un perdido, el otro personaje que muestra un parecido temperamental con el "niño", es también un "enclenque" cuando chico, lo cual se confirma más tarde en la adolescencia.15 Ahora bien: la locura del "niño", con la desorganización de la afectividad primero y de los pensamientos después, la retirada de los demás, el factor celos, la falta de adecuación - que progresa hasta la desconección final-entre las emociones y la expresión de las mismas, es claramente esquizof rénica, sin nota discordante alguna (Lucho, como predispuesto, muestra la misma tendencia del "niño").

13 Para ilustrar hasta qué punto se toma en serio esto del enamoramiento infantil, relataremos una anécdota. Junto con don Eduardo Barrios vive una hija casada y su hijo, el pequeño Leonardo Carvajal Barrios (que aparentaba -en 1960- unos diez u once años de edad), quien tiene una carita sumamente observadora y una actitud tranquila y pensativa (por lo menos cuando aparecía durante las entrevistas). En una ocasión en que el tema tocaba cuestiones que podían sonar algo intrigantes en oídos jóvenes, don Eduardo Barrios, señalando a su atento nieto con los ojos y como dándole extrema importancia, súbitamente exclamó: "¿Cuidado! ¡No olvidarse del "niño"!" (esta palabra en tono de inteligencia, lo que nos hizo ponerla entre comillas) - y a continuación refirió algunos indicios de precocidad sentimental de su nieto. Nos parece que esta anécdota ayuda también a poner en justa perspectiva la que Barrios ha relatado tantas veces de su propio enamoramiento, y al hacerlo, la "objetiviza": no se trata, pues, necesariamente de la experiencia única de un niño raro, que éste mismo, andando el tiempo, trasladó a la literatura, sino de algo que - sin llegar a extremos que interesen a la siquiatría- puede afectar a un niño normal. Sobre este tenue cañamazo Barrios inventó el más complejo, rico y sorprendente bordado con hebras sicológicas, siquiátricas, sociológicas, literarias.

14 El niño que enloqueció de amor ( $6^{\circ} \mathrm{ed}$., Santiago de Chile: Nascimento, 1939), p. 24.

15 Un perdido (Madrid: Espasa-Calpe, 1926), I, 54, 59, II, 36. 
Y para cerrar nuestro círculo de observaciones, resulta que la siquiatría última, desde Kretschmer en I920, ha comprobado que la locura de tipo esquizof rénico ocurre con mucha mayor frecuencia en individuos de constitución alargada y delgada. ${ }^{16}$

Si lo anterior fuese un acierto aislado podría tratarse de una mera coincidencia. Pero ocurre que los aciertos siquiátricos y caracterológicos, siempre nuevos y variados, se repiten en cada párrafo de la novela corta de Barrios, y en cada caso es posible referirlos a investigaciones y hallazgos posteriores en el campo científico. ${ }^{17}$

Lo que acabamos de afirmar, que puede aplicarse a cada rasgo del carácter, así como a la personalidad total del protagonista, alcanza igualmente a los demás personajes, tanto como al caso mismo del "niño" enamorado. El personaje de Barrios no es un engendro tardío de los excesos románticos (como lo son casi todos los versos que inspiró y que corren agregados al final de la novela). Es absolutamente impracticable tratar de entender el caso encandilándose con el amor y dejando en la oscuridad a la locura. Tampoco es permisible ignorar a la madre del niño loco, ni puede dejarse de lado a los otros personajes: esa familia desquiciada que con trazos maestros creó Barrios alrededor del "niño".

16 Edición española, traducción de $1 \mathrm{a} 20^{\mathrm{a}}$ ed. alemana de Körperbau und Charakter - la obra principal de Ernst Kretschmer-con un importante prólogo para España del Dr. J. Solé Sagarra: Constitución y carácter (Barcelona: Labor, 1954). A nosotros nos parece que las últimas ediciones alemanas no aclaran del todo las dudas que se planteaban en las primeras, y por tanto no las superan o suplantan necesariamente. La teoría básica de Kretschmer ha sido criticada por unos pocos, especialmente en los Estados Unidos (v.g.: Allport G. W., Connolly C. J., Garvey R.), y confirmada por muchos estudios en todo el mundo, en particular los especialistas de antropometría como la escuela constitucionalista italiana, y el norteamericano W. H. Sheldon en su obra en colaboración con E. M. Hartl y E. McDermott, Varieties of Delinquent Youtb: an Introduction to Constitutional Psycbiatry (New York: Harper, 1949). La observación a que nos referimos en el texto ya se anticipaba en la obra que juzgamos principal de Sheldon, en colaboración con S. S. Sievens, The Varieties of Temperament: a Psychology of Constitutional Differences (New York: Harper, 1942). Creemos que no corresponde citar página, por cuanto la conclusión a que nos referimos se desprende de la totalidad de las concepciones que se desarrollan en las obras mencionadas, más que de pasajes aislados. En nuestro trabajo más extenso que traeremos a colación en la nota siguiente, hacemos un estudio comparativo en detalle de las observaciones y sistemas de Kretschmer y Sheldon en relación con los personajes de Barrios, y estudiamos aspectos de estos últimos a la luz de otras investigaciones.

17 Véase nuestra: tesis doctoral La verdad sicológica en Eduardo Barrios (Universidad de Minnesota, 1962), donde hemos dedicado unas ciento cincuenta páginas de texto y notas al estudio de El niño que enloqueció de ctmor (parte III). En el capitulo $4^{\circ}$ de la tercera parte, "El primer ensayo caracterológico de Barrios", hemos estudiado los rasgos temperamentales del "niño" y el progreso de su enfermedad, examinando la novela corta de Barrios página por, página $y$ compatándola con los resultados de investigaciones en siquiatría. 
Hacia el final del relato, cualquier lector medianamente atento habrá tenido que reparar en los episodios que delatan la paternidad ilegítima. En realidad basta un solo indicio, a la tercera página del diario, que a la vez sugiere de un toque intenso toda la tragedia del "niño": éste está contemplando desde lejos a "don Carlos"; y dice "me quiere mucho, más que mi mamá" (p. II). En seguida se nos ocurre que es hijo natural o adulterino-no lo sabemos todavía- de Carlos Romeral, pues ¿quién podría querer a un niño más que la propia madre? Y aún el padre, y en una cultura latina... Esa declaración por sí sola, y más la manera ingenua, sin ni siquiera emoción aparente, como de mero hecho que se registra, muestran desde un principio que hay una carencia, un hueco, una herida, en la zona de la afectividad de ese niño. Las páginas que siguen configuran su drama de manera magistral. El amor de Angélica es lo más patente y lo más brillante, aunque no necesariamente lo más patético, emocionante o literariamente bello en el relato. Y no todo es sexo o enamoramiento en la relación "niño"-Angélica, especialmente en el origen de la misma. De igual modo como el chico llena con la persona de Carlos Romeral el hueco en su siquis que corresponde a la falta de padre, sus relaciones con Angélica se integran y juegan en la zona - vacía o lastimada- del afecto maternal. ${ }^{18}$

Cuando apareció esta novela corta, hacía apenas cuatro años que Freud había tenido todavía que corregir por medio de notas, en la tercera edición alemana de La interpretación de los sweños - en la que se describe el complejo de Edipo-, su posición anterior, según la cual los niños están libres de deseos sexuales. No es imposible que Bartios por esa época hubiera oído hablar de Freud, pero es sumamente improbable que lo conociera en detalle cuando muchos siquiatras en todo el mundo todavía lo ignoraban por completo; el reconocimiento internacional de Freud entre especialistas se produce entre los años 1906 y I909. ${ }^{19}$ El lenguaje de Bartios en su conversación, al igual que en sus libros, no contribuye a señalar una influencia freudiana. $Y$ él mismo, Barrios, durante las pláticas que sostuvimos en agosto de 1960 (mencionadas en la nota I I), negó dicha influencia en sus años formativos. Después de la

18 Este análisis se desarrolla en el estudio central de la tercera parte, capítulo $5^{\circ}$ (trabajo citado en la nota anterior): "El medio, los sucesos, las pasiones, en el origen de la locura del 'niño"'.

19 Su biógrafo Ernest Jones afirma que ... el reconocimiento internacional de Freud entre los especialistas es de esos años (The Life and Work of Sigmund Freud, New York: Basic Books, 1953-1957, II, 27), mientras que la fama, y todavía el ablandamiento de importantes resistencias oficiales, empieza en 1926 (Idem., III, 119). 
Primera Guerra Mundial, y más todavía en la década de I930, la crítica empezó a aplicar el complejo de Edipo a los personajes célebres, entre los que no podían faltar Hamlet, Don Juan y Fausto (no nos atrevemos a asegurar que el que resta del cuarteto de grandes, Don Quijote, se haya salvado). Pues bien, aquí tenemos nosotros en español el caso literario al que pueda aplicarse con más lujo de detalles - desenlace esquizof rénico y todo- la línea freudiana de interpretación. La más simple observación de estos elementos hubiera impedido el desconocimiento de la verdad sicológica en la obra de Barrios.

Ni hace falta valernos de Freud para entender al personaje infantil de Barrios, ni es preciso emplear el lenguaje sicoanalítico, que, a nuestro juicio - dicho sea de paso y sin pretensión alguna-es lo más superfluo para un crítico que quiera valerse del sicoanálisis. Que los celos de los niños pequeños pueden ser tan nefastos como los de la gente mayor, es un hecho sabido de las personas perspicaces desde tiempo inmemorial, y no creemos que sea indispensable apuntar a una índole estrictamente sexual (complejo de Edipo) de los celos maternos en el origen de la sicosis del "niño", a pesar del carácter universal que se le atribuye y aparte de la verdad que haya en ello. Pero de todos modos, no es ni siquiera lícito, en el siglo de Freud, hablar de celos de adulto en mente de niño antes de hacer un análisis detenido de los mismos. ${ }^{20}$

La dilucidación de su drama central no agota el contenido de estas páginas preñadas de aciertos de realismo y sicología, de emoción y de bélleza. A modo de resumen, mencionemos en primer término el exten'dido, minucioso y fidelísimo retrato temperamental del "niño", cuyos rasgos, al compararlos a los de los estudios últimos de la personalidad, nunca se contradicen (ver nota $17, y$ obras citadas en la nota 16 -el "niño" revela una "constelación" de rasgos correspondientes a los tempe-

120 Claro es que con Freud pasa lo que puede observarse en los movimientos o autores que se convierten en moda intelectual (como el existencialismo, Marx, Jung, Nietzsche): casi todo el mundo habla como si los conociera y hasta de buena fe cree conocerlos sin haberlos leido, pero muy pocos los aplican oportunamente (a eso se deben, por ejemplo, las gruesas equivocaciones de intelectuales nazis y antinazis con respecto a Nietzsche). En el caso de Freud, el abuso, generalmente vacuo, de la terminología sicoanalítica - que es también una mani. festación de esa moda- ha espantado a más de un estudioso serio, de la consideración de toda crítica que se anuncie como sicológica. Muestras de esa vacuidad de las referencias usuales a Freud pueden encontrarse a cada rato, aun en medios serios y autores intelectuales y de estudio: en el libro de los profesores Orlandi y Ramírez que tenemos a mano (citado en la nota 5), no vemos que se tenga en cuenta a Freud para nada, y sin embargo, a página 14 se lee que la obra Del natural (publicada en 1907) es "hija legitima de Freud y sólo sobrina de Zola", cuando ya vimos que en esos años nadie conocía al siquiatra vienés fuera de sus amistades profesionales (ver nota anterior). 
ramentos esquizotímico de Kretschmer y cerebrotónico de Sheldon). Lo cual se integra con la brillante presentación de la locura, no anunciada desde fuera, sino revelándose por sí sola en su progreso dentro del personaje y en armonía con los rasgos temperamentales a que acabamos

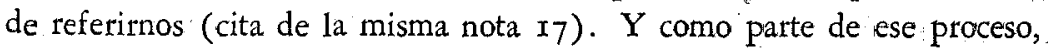
el paso paulatino desde una fantasía exaltada hasta las alucinaciones, $y$ el notable intento de Barrios - que es también un recurso creativo- de. reconocer e'l pensamiento en una fase intermedia entre la expresión poética y la locura, de lo cual no se nos ocurre ningún antecedente literario tal como lo encontramos en esta obra - dentro del pensamiento mismo del perscnaje, y aparte del autor.21 No pasemos por alto el estudia del "rincón" (pp. 69-70), que es todo un documento de su papel en el desarrollo estético de la personalidad. Observemos la tendencia inconsciente al suicidio como recurso desesperado para marcar indeleblemente a la persona amada, y descubramos lo acertado de este rasgo en nuestro personaje. ${ }^{22}$ Demos el lugar que corresponde a la extraordinaria presen-

21 Por ejemplo, ver al protagonista frente al paisaje santiaguino (El niño que enloqueció de amor, p. 62), y compararlo con el episodio delirante de páginas 94-95. La locura no se limita en Barrios a su aparición en esta obra -aunque en las otras no aparezca con su nombre. $Y$ se trata en cada caso de una insanía o anormalidad síquica distinta y congruente-declarada o tan sólo insinuada aparece en Un perdido, "Páginas de un pobre diablo", "Antipatía", El bermano asno, y hasta en Los bombres del bombre (ver nota 82 de nuestra tesis). Es extraño que en general los críticos de Barrios apenas si hayan señalado este aspecto de su creación, en el cual podría colocárselo muy alto en el cuadro de la literatura universal. Raúl Silva Castro, aun cuando su estudio no versa sobre lo sicológico en particular ( $n$ i trata de corrernos con la vaina de terminologías innecesarias, que esconde la vacuidad de más de un crítico), ha descrito con varios detalles acertados la locura de Fray Rufino, en Panorama de la novela chilena (1955, p. 121), y en Historia critica de la novela cbilena (1960, pp. 208-209). Armando Correia Pacheco, en el estudio que preparó para el Diccionario de la literatura latinoamericana - Chile de la Unión Panamericana (1958), ha observado esa "preferencia muy especial por el tema de la locura, principalmente en $E l$ niño que enloqueció de amor (1915) y El bermano asno (1922)". Agreguemos que ésta es también la interpretación del mismo Barrios que corresponde a su complejo personaje de El bermano asno, tal como nos lo manifestó categóricamente en una de las entrevistas a que nos referimos en la nota 12 de este trabajo.

22 El niño que enloqueció de amor, pp. 93-94 y pasajes preparatorios a pp. 39, 40 y 83-84. En el primer pasaje (p. 39) el "niño" se entretiene en imaginarse que el pretendiente de Angélica se suicida, y esto sólo se explica si existe una tendencia suicida en el sujeto pensante. Probablemente pasajes como éste han contribuido a que algunos críticos tacharan de inverosímiles en un preadolescente esas terribles emociones. Un ejemplo de lo acertado que está siempre Barrios: la tendencia al suicidio suele manifestarse en la esquizofrenia infantil, según lo atestigua Lauretta Bender ("Childhood Schizophrenia, Clinical Study of One Hundred Schizophrenic Children", en American Joumal of Orthopsychiatry [1947], XVII, 55). En la tesis citada en la nota 17 desarrollamos este punto, y traemos, entre otras, una correspondencia notable de los recuerdos de la 
tación de los celos, que crecen hasta el paroxismo en la relación con Angélica, aunque de todos modos constituyen otro acierto caracterológico y siquiátrico, $y$ en el "niño" deberían reconocer un origen anterior al episodio de Angélica. ${ }^{23} \mathrm{Y}$ como broche de oro pongamos los pasajes en que se revela su enamoramiento, que llega al deliquio amoroso (pp. 20-2I) $\rightarrow y$ al mismo tiempo un respeto de la inocencia infantil (también realista) que es un encanto más de esta obra de arte.

Además de las consideraciones precedentes, nuestro análisis anterior de los personajes de $U n$ perdid ${ }^{34}$ ha mostrado una riquísima variedad de caracteres, distribuidos polarmente en dos tipos sicológicos generales. El solo hecho de esta bifurcación de caracteres opuestos desmiente la afirmación de excesivo peso de lo autobiográfico y subjetivo justamente en la misma novela que ha sido tildada a veces de autobiográfica, pues ni siquiera en general podría asimilarse al autor a por lo menos uno de los tipos a que pertenecen sus personajes. Aún más, también mostramos que en este aspecto el autor chileno aventaja a varios de los novelistas más famosos "de sicologia", cuyos personajes pertenecen, salvo contadas excepciones, a un solo tipo de personalidad. En el mismo trabajo pusimos de manifiesto el valor científico de las intuiciones de Barrios, que no podrían originarse sin un auténtico y siempre despierto interés en la introspección y en la observación de las sicologías circunstantes - observación directa, inmediata, de la que resultan síntesis valiosas y originales.

Si hemos llegado a estas conclusiones al examinar las obras que más frecuentemente han sido calificadas de subjetivas, cuando no de increí-

adolescencia de Tolstoi (parte III, cap. $4^{\circ}$, pasajes correspondientes a las páginas del texto de Batrios).

23 Tales celos -que, si nuestra visión es válida, reabren y raspan una antigua y profunda llaga de esa alma infantil - y no la prematura excitación sexual o erótica, es lo que precipita el primer acceso violento de locura. En el delirio final Barrios ha puesto sólo dos elementos interpretables, uno de los cuales es el terror de uná aparición monstruosa con rasgos del novio de Angélica sobtepuestos a los de un jabalí de un gobelino que había visto en el comedor de la casa de la muchacha (ver la secuencia de imágenes - progreso de la locura tanto como preparación artística-a pp. 38, 94-5, 99, 106). El otro elemento, el único que queda al final, es el representado por las campanadas de la iglesia -el amor inalterable, desde el principio hasta el fin del diario, por su padre natural "don Carlos". Esta aparición final, en la fantasía esquizofrénica, del pretendiente de Angélica junto al amante de la madre - los rasgos odiosos del primero y todavía el amor e identificación (la "identificación primaria" en términos sicoanalíticos) con el segundo - es una genial intuición sicológica de Eduardo Barrios.

241 "El tipo sicológico en Eduardo Barrios y correspondencias en las letras europeas", Revista iberoamericana, $\mathrm{n}^{\circ} 48$, pp. 264-296. Dicho artículo, ampliado en el número de correspondencias literarias y científicas, así como en las partes de valor interpretativo, forma parte de la tesis citada en la nota 16. 
bles, el estudio de los demás cuentos y novelas de nuestro autor no podrá sino confirmar su punto de partida objetivo, celo científico y genial penetración sicológica. De haber existido algún peligro de malogro en la creación de Barrios, creemos que hubiera sido precisamente la tentación de poner demasiada ciencia, de dejar transparentarse - si no exponer-parte del tremendo caudal de observaciones e intuiciones sicológicas. ${ }^{25}$ No pocas páginas, y no pocas novelas, aun entre las de más prestigio (pensemos en otro novelista en español, Baroja, gran observador como el chileno), se malogran artísticamente a causa de no haber sabido el autor resistir aquel impulso o deseo de decir algo de lo que sabe. Y, paradójicamente, este "subjetivismo" de la actitud del novelista - este dejarse ver en desmedro de los personajes - suele producir una especie de "objetivismo" en un plano único superficial, que en sus mejores momentos cubre una gama que va desde lo erudito hasta lo periodístico, mientras que en los peores revela la ambición frustrada del sabio a medias.

Justamente el celo extremado de Barrios por no viciar su obra lo llevó a su concepción del arte y de la estructuración creativa en tres planos, y a sujetarse estrictamente a la misma. Es la materia de vida y verdad de que se valió, más esa rica y acabada elaboración en sentido vertical, lo que constituye la cualidad más saliente de su obra - la cualidad que establece su título a un lugar destacado y estable en la literatura universal.

ANgel Manuel VázQuez-Bigi

Northern Illinois University.

$25 \mathrm{Y}$, en efecto, en $U_{n}$ perdido se le escapan unas pocas frases que están de más en novela (ver nota 14 de nuestro artículo, que se amplió en la 28 de la tesis, por ejemplo). Por suerte son muy pocas (y nos facilitaron nuestra visión critica). 
